\section{Characterization of Chromosomal Aberrations in Neuroblastoma Formalin-Fixed Paraffin- Embedded Specimens with Standard ArrayCGH Procedure - Preliminary Experience}

\section{Abstract}

Background: The broad spectrum of neuroblastoma (NB) clinical behavior depends on a genomic landscape of tumor cells. The amplification of MYCN oncogene is the most powerful negative prognostic marker in NB. Moreover, segmental chromosomal alterations are also associated with a poor outcome. Therefore, the comprehensive characterization of tumor genetic features is obligatory for NB patients. These features determine the risk stratification and therapeutic decisions in treatment.

Purpose: Our report focuses on a possibility to use standard microarray procedure to demonstrate critical structural chromosomal alteration in archival samples of NB tumors.

Methods and Findings: Formalin-fixed paraffin-embedded tissue samples from 8 NB primary tumors have been analyzed by cytogenetic microarrays. It achieved a very good quality of genomic DNA from fixed samples. Chromosomal abnormalities were detected in 7 out of 8 cases. It was not an incidence of MYCN amplification.

Conclusion: The results demonstrate that it is possible to obtain reliable and highquality microarray data from archival samples.

Keywords: Advanced neuroblastoma; Chromosomal aberrations; Molecular karyotyping; Tumor genome screening

\section{Katarzyna Szewczyk* \\ Department of Medical Genetics, Chair of Pediatrics, Faculty of Medicine, Jagiellonian University Medical College, Krakow, Poland}

*Corresponding author: Katarzyna Szewczyk

= katarzyna.szewczyk@uj.edu.pl

Department of Medical Genetics, Chair of Pediatrics, Faculty of Medicine, Jagiellonian University Medical College, Krakow, Poland.

Tel: 48-12-658-20-11, ext.1296

\section{Citation: Szewczyk K (2017)}

Characterization of Chromosomal Aberrations in Neuroblastoma FormalinFixed Paraffin-Embedded Specimens with Standard ArrayCGH Procedure - Preliminary Experience. Transl Biomed. Vol. 8 No. 3: 117.

Received: June 26, 2017; Accepted: July 02, 2017; Published: July 06, 2017

\section{Introduction}

Neuroblastoma (NB) is one of the most common pediatric cancer (6\% to $10 \%$ ) [1]. Approximately $90 \%$ of patients are diagnosed before the age of 5 years old [2,3]. The primary form of NB is an extracranial solid tumor arisen from neural crest progenitor cells [1-4]. NB tumors have a very high tendency to form distant metastases [2]. The comprehensive characterization of tumor genetic and biological features is obligatory for NB patients [4]. These features determine the risk stratification and therapeutic decisions in treatment. The most unfavorable genetic factor in NB is MYCN oncogene amplification [2-7]. Moreover, tumor aggressiveness and progression is strongly associated with Segmental Chromosomal Aberrations (SCAs) observed in malignant cells $[2,5,8]$. Aggressive metastatic tumors are characterized by a high number of SCAs and a low number of
Numerical Changes (NCAs). On the whole, genome-wide studies have demonstrated that critical chromosomal damages occur more frequently in older patients and that SCAs accumulate in an age-dependent manner, as supported by Schleiermacher and Coco [8-12].

According to the international guidelines the current gold standard for detecting MYCN oncogene amplification in NB tumor cells is the fluorescence in situ hybridization (FISH) analysis of interphase nuclei [5]. Other, critical copy number changes like $11 q 23$ deletion and $17 q$ gain can also be evaluated by FISH analysis [5,13-17]. However, pan-genomic techniques like highresolution array-based comparative genomic hybridization (aCGH) or multiplex Ligation-Dependent Probe Amplification (MLPA) provide alternative methods for whole-genome screening and characterizing SCAs and NCAs in NB tumor cells [18-21]. These techniques are equivalent to thousands of FISH 
experiments. Numerous aCGH studies had performed on NB primary tumor tissues revealed new, often occurring unfavorable SCAs including deletions in regions: $3 p, 4 p, 6 q, 10 q, 14 q, 18 q$, and additional copies: $3 p, 12,18 p$, as well as cases of the uniparental disomy and the chromothripsis in malignant cells $[8,17,21-25]$.

The vast majority of FISH and aCGH routine analysis in NB have been and currently are performed on fresh tumor tissue samples. However, it is worth emphasizing that both research techniques provide for a genetic evaluation also formalin-fixed paraffinembedded (FFPE) specimens. Archival, FFPE samples represent an invaluable source of material especially for retrospective molecular studies. The possibility of long-term clinical follow-up of FFPE samples makes them a valuable source to evaluate links between genetic and clinical information.

So far, a few studies have used FFPE-derived DNA for aCGH due to nucleic acid degradation and chemical modifications, and also cross-linking with proteins introduced during the fixation process. In NB, recently reported study confirmed an ability to recognize chromosomal aberrations in FFPE specimens using aCGH but with a new modified microarray method (OncoScan ${ }^{\mathrm{TM}}$ FFPE Assay Kit, Affymetrix) [26]. The used protocol gave rise to a significant improvement over most of the previous methods in terms of accuracy in detecting copy number variant in FFPE tissues.

The main purpose of this study is evaluation the practical potential of routine use aCGH platform for detection of numerical and structural chromosomal changes in FFPE-derived DNA samples of NB primary tumor tissues.

\section{Material and Methods}

Diagnostic fixed tissue samples (FFPE specimens) from NB primary tumors have been analyzed in Laboratory of Molecular Genetics, University Children's Hospital in Krakow since June 2016. FFPE tissues required initial de-waxed with xylene followed by washing with ethanol. Genomic DNA was extracted from the sample with use of a DNA tissue kit (KURABO Industries Ltd, Osaka, JAP) and quantified by OD at $260 \mathrm{~nm}$ using the NanoDrop ND-1000 (NanoDrop Technologies, Inc., Wilmington, DE). A total of $350 \mathrm{ng}$ of DNA was used for analysis by means of $60 \mathrm{~Kb}$ cytogenetic microarrays (SurePrint G3 CGH ISCA v2 8x60K, Agilent Technologies, Santa Clara, CA), according to the manufacturer's protocols.

Chromosomal alterations were identified by dedicated software (CytoGenomics 2.9.2.4., Agilent Technologies, Santa Clara,
CA) and manually verified by the author. It was established a detection limit of $200 \mathrm{~kb}$ for appropriate detection of structural chromosomal abnormalities.

\section{Results}

The aCGH evaluation was performed for 8 FFPE specimens. It was achieved a very good quality of genomic DNA with the described isolation kit. Moreover, there were no obstacles to achieving the required DNA quantity.

Abnormalities in the form of numerous NCAs were detected in 5 out of 8 cases. These tumors were identified as hyperdiploid. The most common NCAs were multiplication of whole chromosomes: $6,7,8,9,17,20,22$. In 2 cases we have found also loss of whole chromosomes (Table 1). All patients with the NCAs-positive aCGH profile were younger than 18 months.

Both, SCAs and NCAs were found in 2 out of 8 cases (Figure 1). These patients were older than 18 months, what was an unfavorable factor. Among SCAs we have found critical changes like 1p36 loss and $17 q$ gain. The size of above structural abnormalities ranged from $5.8 \mathrm{Mb}$ to $49.5 \mathrm{Mb}$. It was not an incidence of MYCN amplification (Table 1). One patient from the research group had a normal diploid aCGH profile, and was over 18-months-old.

\section{Discussion}

There is a well-known association between patient outcome and tumor ploidy in NB [2-5]. NB tumors with diploid DNA content are more likely to present in older children and with metastatic disease. These diploid tumor's cells are more likely to harbor MYCN gene amplification or other SCAs. In contrast, neartriploid DNA content is most commonly found in infant tumors that remain localized and are associated with more favorable outcome $[2-9,12]$.

The most common type of SCAs in NB cells beside MYCN amplification is gain of the distal part of long arm of chromosome 17 which occurs in at least $50 \%$ of primary NB tumors. This abnormality is associated with poor prognosis, and is frequently coexisted with other biomarkers of aggressive disease such as MYCN amplification, older age, and chromosome 1p36 deletion. Loss of 1 p36 region occurs in approximately a quarter of NB tumors and also predicted adverse outcome $[2,3,7,11,13]$. In addition, a recent INRG report on non-MYCN amplified tumors determined that it is not single genetic markers, but the overall

Table 1 Microarray analysis results in the research group.

\begin{tabular}{|c|c|c|}
$\begin{array}{c}\text { Patient } \\
\text { number }\end{array}$ & $\begin{array}{c}\text { Patient age } \\
\text { (months) }\end{array}$ & \multicolumn{1}{c}{ Abnormal acGH profile } \\
\hline 1 & $>18$ & $\operatorname{arr}[\mathrm{hg} 19] 1 \mathrm{p} 36.13 \mathrm{p} 33 \times 1,17 \times 3,18 \times 3,19 \mathrm{p} 13 \mathrm{p} 11 \times 1$ \\
\hline 2 & $>18$ & $\operatorname{arr}[\mathrm{hg} 19] 1 \mathrm{p} 36.33 \mathrm{p} 36.31 \times 1,3 \times 1,4 \times 1,6 \times 1,7 \times 3,9 \times 1,10 \times 1,14 \times 1,15 \times 1,16 \times 1,17 q 21.2 q 25.3 \times 3,19 \times 1,21 \times 1,22 \times 1$ \\
\hline 3 & $<18$ & $\operatorname{arr}[\mathrm{hg} 19] 2 \times 3,6 \times 3,7 \times 3,8 \times 3,13 \times 3,17 \times 3,18 \times 3,20 \times 3$ \\
\hline 4 & $<18$ & $\operatorname{arr}[\mathrm{hg} 19] 3 \times 1,6 \times 3,7 \times 3,9 \times 3,16 \times 1,17 \times 3,20 \times 3,21 \times 3$ \\
\hline 5 & $<18$ & $\operatorname{arr}[\mathrm{hg} 19] 6 \times 3,7 \times 3,8 \times 3,9 \times 3,13 \times 3,17 \times 3,20 \times 3,21 \times 1,22 \times 3$ \\
\hline 6 & $<18$ & $\operatorname{arr}[\mathrm{hg} 19] 1 \times 3,2 \times 3,5 \times 3,6 \times 3,7 \times 3,12 \times 3,13 \times 3,17 \times 3,20 \times 3,22 \times 3$ \\
\hline 7 & $<18$ & $\operatorname{arr}[\mathrm{hg} 19] 1 \times 3,2 \times 3,6 \times 3,7 \times 3,8 \times 3,9 \times 3,12 \times 3,17 \times 3,19 \times 3,20 \times 3,22 \times 3$ \\
\hline
\end{tabular}



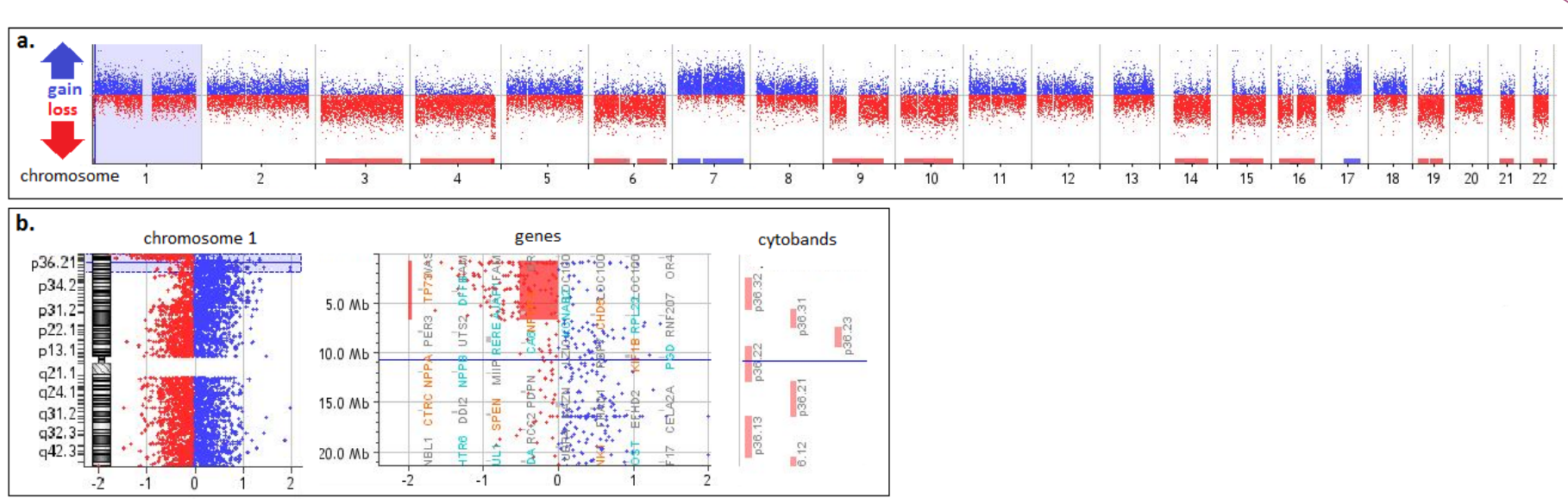

Figure 1 Microarray profile for NB tumor. a) Segmental and numerical changes; b) Zoom in deletion of chromosome $1 p 36$.

segmental genomic profile of tumors that adds information to patient prognosis [11].

\section{Conclusion}

Whole-genome DNA copy number analyses have demonstrated that the overall genomic pattern adds significant prognostic information to clinical algorithms. Integration of molecular profiles and genomic markers that are reflective of tumor biology into future classification systems will allow more precise prognostication and refined treatment assignment.

\section{References}

1 Hallett A, Traunecker H (2012) A review and update on neuroblastoma. Paediatr Child Health 22: 103-107.

2 Louis CU, Shohet JM (2015) Neuroblastoma: Molecular pathogenesis and therapy. Annu Rev Med. 66: 49-63.

3 Van Roy N, De Preter K, Hoebeeck J (2009) The emerging molecular pathogenesis of neuroblastoma: Implications for improved risk assessment and targeted therapy. Genome Med 1: 74.

4 Ambros PF, Ambros IM (2001) SIOP Europe neuroblastoma pathology, biology, and bone marrow group. Pathology and biology guidelines for resectable and unresectable neuroblastic tumors and bone marrow examination guidelines. Med Pediatr Oncol 37: 492-504.

5 Ambros PF, Ambros IM, Brodeur GM (2009) International consensus for neuroblastoma molecular diagnostics: A report from the International Neuroblastoma Risk Group (INRG) biology committee. Br J Cancer 100: 1471-1482.

6 Canete A, Gerrard M, Rubie H (2009) Poor survival for infants with MYCN-amplified metastatic neuroblastoma despite intensified treatment: The International Society of Paediatric Oncology European Neuroblastoma Experience. J Clin Oncol 27: 1014-1019.

7 Janoueix-Lerosey I, Schleiermacher G, Michels E (2009) Overall genomic pattern is a predictor of outcome in neuroblastoma. J Clin Oncol 27: 1026-1033.

8 Schleiermacher G, Janoueix-Lerosey I, Delattre O (2014) Recent insights into the biology of neuroblastoma. Int J Cancer 135: 2249-2261.
Archival samples like FFPE specimens are an excellent source of DNA for aCGH processing with the advantage of simple handling, distribution and storage of tumors tissue. Moreover, aCGH analysis is cost-effective technology gives an opportunity to comprehensive characterization of neoplastic genome. In conclusion, we believe that molecular karyotyping with use of standard-resolution cytogenetic microarrays can be recommended as a valuable method for detection of chromosomal aberrations in archival fixed samples of NB and other solid tumors.

9 Schleiermacher G, Janoueix-Lerosey I, Ribeiro A (2010) Accumulation of segmental alterations determines progression in neuroblastoma. J Clin Oncol 28: 3122-3130.

10 Schleiermacher G, Michon J, Ribeiro A (2011) Segmental chromosomal alterations lead to a higher risk of relapse in infants with MYCN-non-amplified localised unresectable/disseminated neuroblastoma (a SIOPEN collaborative study). Br J Cancer 105: 1940-1948.

11 Schleiermacher G, Mosseri V, London WB (2012) Segmental chromosomal alterations have prognostic impact in neuroblastoma: A report from the INRG project. Br J Cancer 107: 1418-1422.

12 Coco S, Theissen J, Scaruffi P (2012) Age-dependent accumulation of genomic aberrations and deregulation of cell cycle and telomerase genes in metastatic neuroblastoma. Int J Cancer 131: 1591-1600.

13 Abel F, Ejeskär K, Kogner P (1999) Gain of chromosome arm 17q is associated with unfavourable prognosis in neuroblastoma, but does not involve mutations in the somatostatin receptor 2 (SSTR2) gene at 17q24. Br J Cancer 81: 1402-1409.

14 Stallings RL, Carty P, McArdle L (2004) Molecular cytogenetic analysis of recurrent unbalanced $t(11 ; 17)$ in neuroblastoma. Cancer Genet Cytogenet 154: 44-51.

15 Villamón E, Berbegall AP, Piqueras M (2013) Genetic instability and intratumoral heterogeneity in neuroblastoma with MYCN amplification plus 11q deletion. PLoS One 8: e53740.

16 Carén H, Kryh H, Nethander M (2010) High-risk neuroblastoma tumors with 11q-deletion display a poor prognostic, chromosome 
instabilityphenotype with later onset. Proc Natl Acad Sci USA 107: 4323-4328.

17 Lundberg G, Jin Y, Sehic D (2013) Intratumor diversity of chromosome copy numbers in neuroblastoma mediated by on-going chromosome loss from a polyploid state. PLoS One 8: e59268.

18 Combaret V, lacono I, Bréjon S (2012) Analysis of genomic alterations in neuroblastoma by multiplex ligation-dependent probe amplification and array comparative genomic hybridization: A comparison of results. Cancer Genet 205: 657-664.

19 Tumer S, Altungoz O, Bagci O (2016) The detection of genetic parameters for prognostic stratification of neuroblastoma using multiplex ligation-dependent probe amplification technique. Genet Test Mol Biomarkers 20: 74-80.

20 Ambros IM, Brunner B, Aigner G (2011) A multilocus technique for risk evaluation of patients with neuroblastoma. Clin Cancer Res 17: 792-804.

21 Carén H, Erichsen J, Olsson L (2008) High-resolution array copy number analyses for detection of deletion, gain, amplification and copy-neutral LOH in primary neuroblastoma tumors: Four cases of homozygous deletions of the CDKN2A gene. BMC Genomics 9: 353.

22 Khan FH, Pandian V, Ramraj S (2015) Acquired genetic alterations in tumor cells dictate the development of high-risk neuroblastoma and clinical outcomes. BMC Cancer 15: 514.

23 Villamón E, Piqueras M, Mackintosh C (2008) Comparison of different techniques for the detection of genetic risk-identifying chromosomal gains and lossesin neuroblastoma. Virchows Arch 453: 47-55.

24 Abbasi MR, Rifatbegovic F, Brunner C (2015) Bone marrows from neuroblastoma patients: An excellent source for tumor genome analyses. Mol Oncol 9: 545-554.

25 Bogen D, Brunner C, Walder D (2016) The genetic tumor background is an important determinant for heterogeneous MYCN-amplified neuroblastoma. Int J Cancer 139: 153-163.

26 Pinto N, Mayfield JR, Raca G (2016) Segmental chromosomal aberrations in localized neuroblastoma can be detected in formalinfixed paraffin-embedded tissue samples and are associated with recurrence. Pediatr Blood Cancer 63: 1019-1023. 